Int. J. Dev. Biol. 50: 399-403 (2006)

doi: $10.1387 / \mathrm{ijdb} .052029 \mathrm{sz}$

Original Article

\title{
Cloning, expression and functional study of translation elongation factor 2 (EF-2) in zebrafish
}

\author{
SHU-HONG ZHANG", JI-HUA YAO\#,*, HUAI-DONG SONG ${ }^{1}$, LU WANG and JING-LUN XUE* \\ State Key Laboratory of Genetic Engineering, Institute of Genetics, School of Life Sciences, Fudan University, Shanghai, China and \\ ${ }^{1}$ Center of Molecular Medicine and Human Genome, Ruijin Hospital, Shanghai Second Medical University, Shanghai, China
}

\begin{abstract}
We have identified translation elongation factor 2 (EF-2) in zebrafish (GenBank Accession No. AA091234). Analysis of the DNA sequence of zebrafish EF-2 shows that the 2826 bp cDNA spans an open reading frame between nucleotide 55 to 2631 and encodes a protein of 858 amino acids. Zebrafish EF-2 protein shares 92\%, 93\%, 93\% and $92 \%$ identity with the corresponding amino acid sequence in human, mouse, Chinese hamster and Gallus EF-2, respectively. Whole-mount in situ hybridization showed that zebrafish EF-2 was a developmentally regulated gene and might play important roles during the early development of zebrafish embryos. Therefore, we further studied the function of EF-2 during early embryogenesis. Using morpholino antisense oligo knockdown assays, anti-MO injected embryos were found to display abnormal development. The yolk balls were larger than normal and the melanophores spreading on their bodies became fewer. Furthermore, their tails were incurvate and their lenses were much smaller than those of the normal embryos. However the EF-2 overexpression data showed that extra EF-2 protein had no obvious effect on zebrafish embryonic development.
\end{abstract}

KEY WORDS: EF-2, zebrafish, whole-mount in situ hybridization, overexpression, knockdown

\section{Introduction}

Translation elongation factor 2, EF-2 and its eubacterial homolog, EF-G, act sequentially after eEF1a/EF-Tu to catalyze the GTP hydrolysis-dependent translocation of the ribosome during protein synthesis, to allow the peptidyl-tRNA to move from aminoacyl site to the peptidyl site on a ribosome, liberating the former site to accept a new mRNA triplet and its cognate ternary complex (eEF1-aminoacyl-tRNA-GTP) (Kohno K et al., 1986; Mendoza A et al., 1999). EF-2 is a GTPase; it binds and hydrolyzes GTP and forms a ternary complex with GTP and ribosomes (Rao S et al., 1996).

Although regulation of EF-2 is well known to include phosphorylation and ADP-ribosylation, developmental regulation of $E F-2$ isoforms has not been fully explored. The reported developmentally regulated EF-2 isoforms constitute a family of genes in Tetrahymena thermophila, which encode proteins homologous to EF-2 and are expressed only during sexual reproduction (Malave TM et al., 2004). Drosophila contains two nearly identical EF-2 genes (Lasko, 2000). Nevertheless, there are many examples in multicellular eukaryotes of differential expression of isoforms of translation elongation factor 1a (eEF-1a). Drosophila has two copies ( $F 1$ and $F 2$ ) of the EF-la gene, which are expressed at different times during development. While $F 2$ is transcribed only in the pupal stage, $F 1$ is a housekeeping gene expressed in all cells during development and in adulthood (Hovemann B et al., 1988). Three EF-lagenes have been characterized in Xenopus (42Sp50, EF-laO and EF-laS), 42Sp50 is expressed exclusively in oocytes, while $E F$-laOis active from fertilization until early onset of neurulation (Krieg $\mathrm{P}$ et al., 1989; Dje MK et al., 1990). In mammals, the EF-S (eEF1a2) gene is expressed in heart and muscle tissue and eventually becomes the only form expressed in these tissues (Knudsen et al., 1993). Disruption of the EF-S (eEF1a2) gene in mouse results in deficiencies in muscle and neuronal function within a few weeks after birth (Chambers et al., 1998).

Since zebrafish embryos develop externally and are optically transparent, this system may cast light on the function of EF-2. In this report, we cloned zebrafish EF-2 gene and examined its

Abbreviations used in this paper: EF-2, translation elongation factor 2; MO, morpholino modified antisense oligonucleotide; PTU, 1-phenyl-2-thiourea; PFA, paraformaldehyde; ORF, open reading frame; hpf, hours postfertilization.

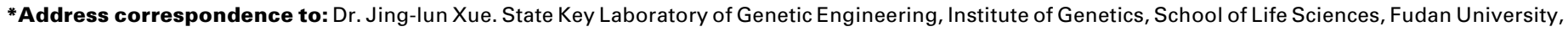
Shanghai 200433, China.Fax: +86-21-6564-9899. e-mail: jlxue@fudan.edu.cn or yaojh@fudan.edu.cn (Dr. Yao).
}

\#Note Both authors contributed equally to this paper. 

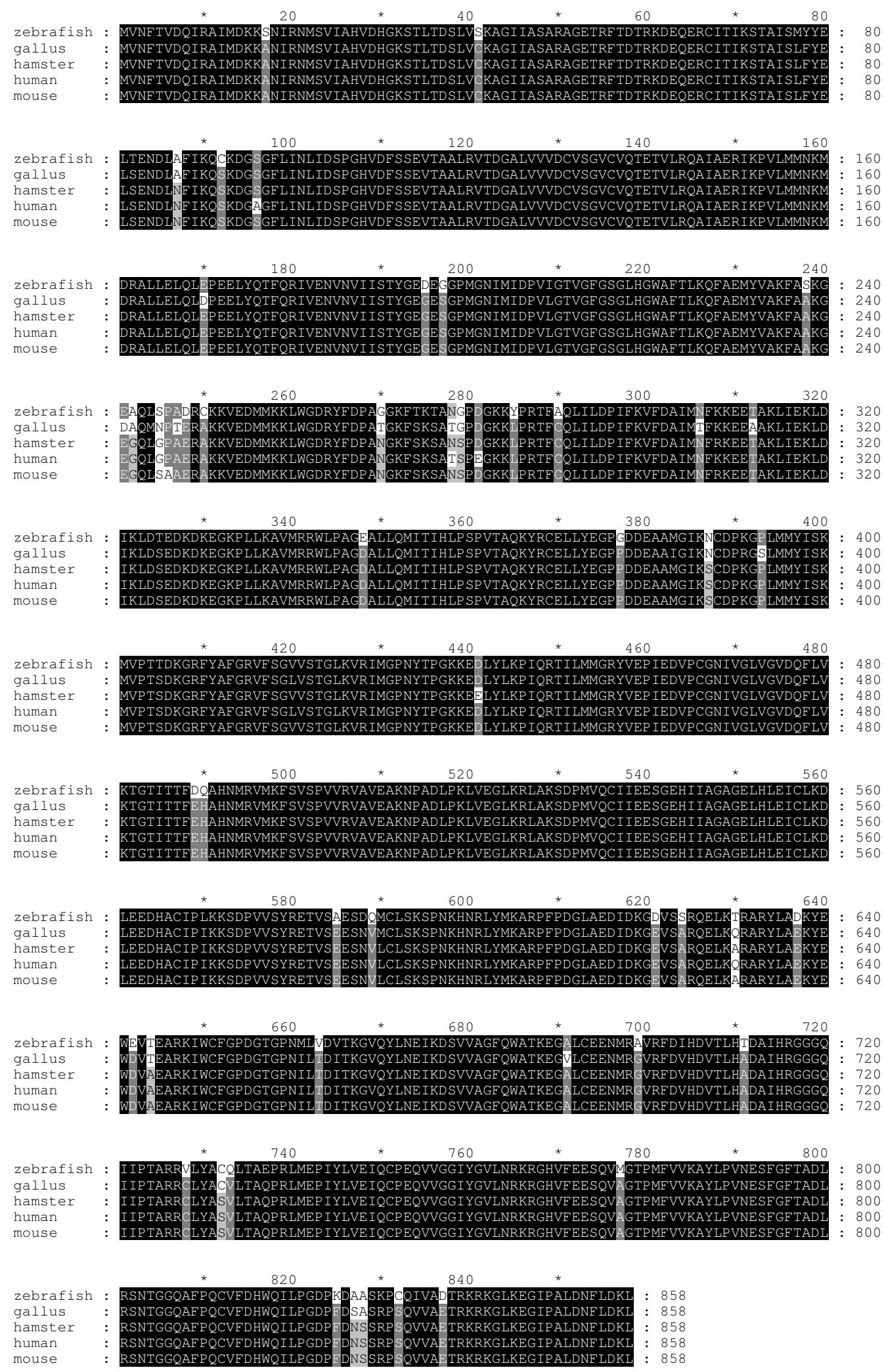

Fig. 1. Alignments of zebrafish, Chinese hamster, Gallus gallus, human and mouse EF-2. Amino acids are shaded according to the degree of conservation using GeneDoc: black (100\% similarity), gray (80\% similarity) and light gray (60\% similarity). The amino acid sequences of Chinese hamster, Gallus gallus, Human and Mouse EF-2 were obtained from the GenBank database with Accession Nos. A25440, NP-990699, CAA77750 and NP-031933, respectively. 
A

B

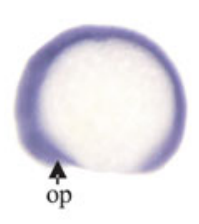

C

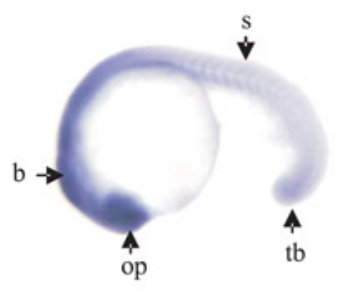

$\mathbf{E}$

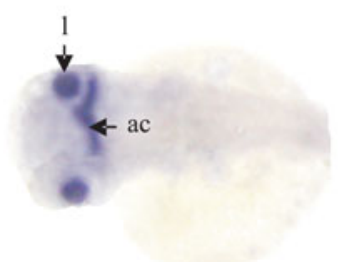

D

$\mathbf{F}$
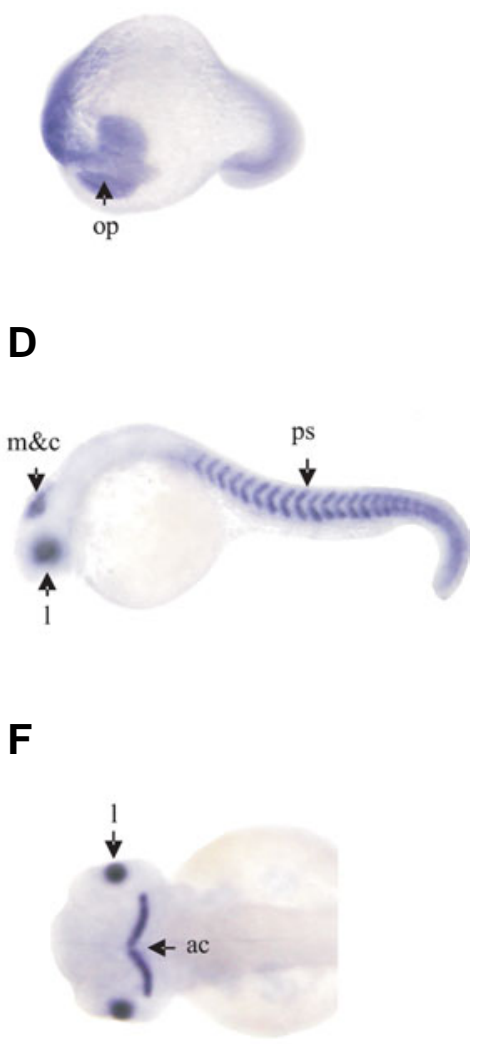

Fig. 2. Expression patterns of EF-2 in the zebrafish embryo. Side view $(A, B, C, D)$; dorsal view (E,F).(A) 5-somite stage; (B) 17-somite stage; (C) 22 -somite stage; (D) prim-15 stage; (E) prim-25 stage; (F)protrudingmouth stage. Abbreviations: op, optic primordial; $b$, brain; tb, tail bud; $s$, somite; $m \& c$, midbrain and cerebellum; I, lens; ps, posterior somites; ac, anterior portion of the cerebellum.

expression pattern by whole-mount in situ hybridization. The result of whole-mount in situ hybridization showed that zebrafish $E F-2$ was a developmentally regulated gene just like EF-S and might play important roles during the early development of zebrafish embryos. Therefore, we further studied the function of EF-2 during early embryogenesis.

\section{Results}

\section{Sequence analysis of the zebrafish EF-2 gene}

The full-length zebrafish EF-2 gene was sequenced. The result shows that the 2826 bp cDNA spans an ORF between nucleotide 55 to 2631 and encodes a protein of 858 amino acids (GenBank Accession No. AAQ91234).

To figure out the structural features of the zebrafish EF-2 protein, its amino acid sequence was compared with those of other EF-2 proteins using clustalw version 1.82 online (http:// www.ebi.ac.uk/clustalw/). The zebrafish EF-2 protein is highly conserved sharing identity with overall $92 \%, 93 \%, 93 \%$ and $92 \%$ of amino acid sequence in human (CAA77750), mouse (NP_031933), Chinese hamster (A25440) and Gallus gallus (NP_990699) EF-2, respectively. Then the alignment was done

with software GeneDoc (shown in Fig. 1). The data shows that the zebrafish EF-2 protein is highly conserved.

\section{Spatiotemporal expression pattern of the zebrafish EF-2 gene}

EF-2 transcript was not detected during somitogenesis using sense probe (data not shown). However, the results of wholemount in situ hybridization using antisense probe showed that a large amount of EF-2 transcripts existed during somitogenesis. EF-2 transcripts originally appeared strongly throughout envelope at 5-somite stage (Fig. 2A). At 17-somite stage (Fig. 2B) and 22-somite stage (Fig. 2C), EF-2 transcripts were detected still throughout the embryo with especially significant in the optic primordia, the whole brain, the trunk and tail bud. From 22-somite stage the stained cells began to concentrate strongly in the eyes, brain and somites. At prim-15 stage, the stained cells concentrated mainly in the lens, retina, midbrain and cerebellum (Fig. 2D) and posterior somites expression is especially evident. But after prim-25 stage the expression of $E F-2$ only appeared in the lens and the anterior portion of the cerebellum (including the proliferation zone at the midbrainhindbrain - boundary) (Fig. 2 E-F).

\section{Overexpression of EF-2}

Expressing vector pcDNA3.0-EF2 with various amounts (1.0ng/embryo, $0.67 \mathrm{ng} / \mathrm{embryo}$ and $0.33 \mathrm{ng} / \mathrm{embryo}$ ) and $E F-2$ capped mRNA with the amount of $4.5 \mathrm{ng} /$ embryo were injected into each of 1-4-cell-stage embryos, respectively. After injecting pcDNA3.0-EF2 expression vector with the amount of $1.0 \mathrm{ng} /$ embryo, most of the zebrafish embryos died (Table 1), showing that the amount of $1.0 \mathrm{ng} /$ embryo was fatal to the zebrafish embryos. However, no abnormal phenotypes appeared when they were injected by other amounts of pcDNA3.0-EF2 (0.67 ng/embryo and $0.53 \mathrm{ng} / \mathrm{embryo}$ ) or EF-2 capped mRNA (Table 1 ). So, overexpression of $E F-2$ had no obvious effect on the zebrafish embryonic development (Fig. 3).

\section{Gene knockdown with morpholino antisense oligo}

The results of gene knockdown assay with morpholino antisense oligo showed that most embryos injected with antiMO displayed abnormal phenotypes during embryonic development while the same dose of sense-MO had no obvious effect on the embryos (Table 1). From 24-hpf EF-2-MO-treated embryos began to show developmental growth arrest. The EF2-MO treated embryos were shorter, yolk balls were larger, tails were incurvate, lenses were much smaller and the melano-

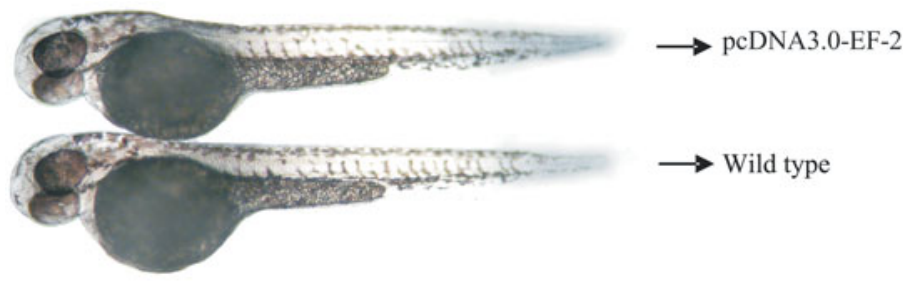

Fig. 3. Overexpression of the $\mathbf{E F - 2}$ gene (48 $\mathbf{~ h p f ) . ~ T h e r e ~ i s ~ n o ~ o b v i o u s ~}$ difference between the pcDNA3.0-EF2 injected zebrafish and the wild type zebrafish. 
A

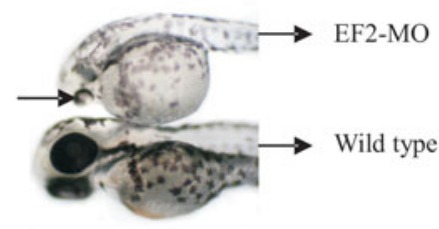

C

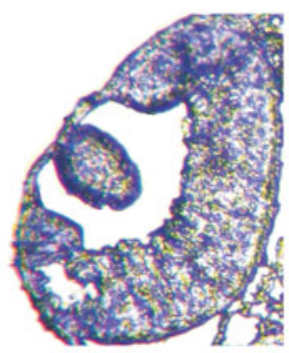

B

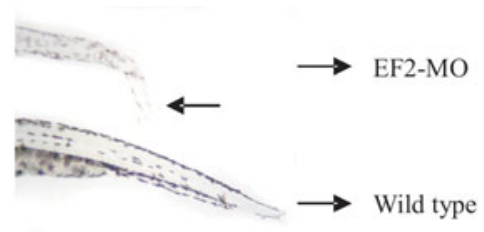

D

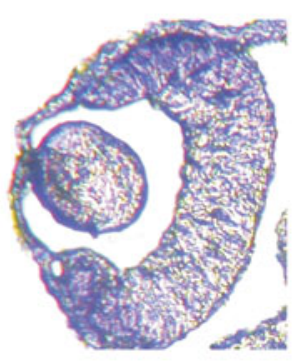

E

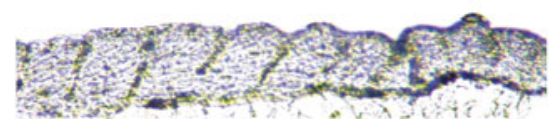

$\mathbf{F}$

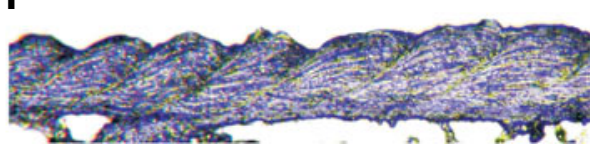

Fig. 4. Morphological and histological analysis of EF2-MO injected embryos at $\mathbf{4 8} \mathbf{~ h p f . ~}$ (A,B) Morphological comparisons of EF2-MO injected embryos and control embryos. (C,D) Histological analysis of EF2-MO injected embryos through cross-sections. (E,F) The crosssections of sense-MO injected embryos. It is obvious that in the EF-2-MO injected embryo, the lens is much smaller and the tail is incurvate and incompact.

phore spreading on their bodies became lighter than normal level (Fig. 4 A,B). Furthermore cross-sections also revealed that their tails were incurvate and their lenses were much smaller than those of normal embryos (Fig. 4 C-F).

\section{Discussion}

We have identified a translation elongation factor $2(E F-2)$ in zebrafish with high identity to EF-2 proteins of other animals. From the results of the whole-mount in situhybridization, it can be presumed that the zebrafish EF-2 gene is developmentally regulated. Because zebrafish $E F-2$ transcripts appear strongly

TABLE 1

\section{OVEREXPRESSION AND MORPHOLINO KNOCKDOWN OF THE EF-2 GENE}

\begin{tabular}{lcccc} 
& $\begin{array}{c}\text { Amount of } \\
\text { microinjection } \\
\text { (ng/embryo) }\end{array}$ & $\begin{array}{c}\text { Whole mount } \\
\text { embryos }\end{array}$ & $\begin{array}{c}\text { Live embryos } \\
\text { (\%) }\end{array}$ & $\begin{array}{c}\text { Injected and phenotype } \\
\text { for over-expression } \\
\text { studies (\%) }\end{array}$ \\
\hline pcDNA3.0-EF2 & 1.0 & 263 & $12(4.6)$ & $2(16.7)$ \\
& 0 & 88 & $59(67.0)$ & $0(0)$ \\
& 0.67 & 248 & $120(48.4)$ & $7(5.8)$ \\
& 0 & 60 & $39(65.0)$ & $0(0)$ \\
EF-2 capped mRNA & 0.53 & 169 & $83(49.1)$ & $3(3.6)$ \\
& 0 & 64 & $37(57.8)$ & $0(0)$ \\
EF-2 morpholino & 4.5 & 211 & $126(59.7)$ & $7(5.6)$ \\
& 0 & 73 & $48(65.8)$ & $0(0)$ \\
control sense-MO & 4.5 & 116 & $87(75)$ & $52(60)$ \\
& 0 & 96 & $78(82)$ & $0(0)$ \\
& 0 & 167 & $130(77.8)$ & $0(0)$ \\
\hline
\end{tabular}
iary role. throughout envelope before prim-15 stage, we consider the EF-2 gene is expressed as a maternal transcript. After prim-15 stage, the expression of zebrafish EF-2 is limited to irreversibly growth-arrested cells such as lens, cerebellum and somites, so we think zebrafish EF-2 is a terminal differentiation-specific protein just like EF-S protein (Knudsen et al., 1993; Chambers et al., 1998). And we can also accordingly presume that zebrafish EF-2is potentially a multi-gene family and the one which we are reporting is only one isoform of this family and its main function is maintaining the development of lens, cerebellum and somites but not protein synthsis.

This seems to be the first functional report on the developmental regulation of zebrafish EF-2 gene. Overexpression of EF-2 had no obvious effect on the zebrafish embryonic development. The data of gene knockdown approved that EF2surely plays important roles in maintaining the development of zebrafish embryos. As EF-2 proteins are expressed in posterior somites and the tail bud, so in the EF-2-MO treated embryos the low level of EF-2 could not maintain the production of new somites in the tail buds and finally result in incurvate tails. Similarly, EF-2 proteins are also expressed in the lenses and were important to maintain their development, so probably hypoplasia appeared at those places when the level of the EF-2 could not maintain normal lens development. Although EF-2 expressed in the anterior portion of the cerebellum, no obvious abnormalities were found here upon the treatments, so we presume that some other important genes participate in the development of the anterior portion of the cerebellum and EF-2 only had a subsid-

Above all, from the results of the whole-mount in situhybridization, overexpression and gene "knock-down" experiments, we can believe that the zebrafish EF-2gene regulates primarily specific aspects of development.

\section{Materials and Methods}

\section{Zebrafish and embryo maintenance}

Zebrafish were raised and maintained under standard laboratory conditions at $28^{\circ} \mathrm{C}$, as described by Westerfield et al. (Westerfield et al., 1995). Embryos used in whole-mount in situ hybridization were raised in $0.003 \%$ PTU (1-phenyl-2-thiourea, Sigma, St.Louis, MO, USA) to prevent pigment formation. The stage of the embryos was determined by morphological features and fixed with 4\% PFA (paraformaldehyde) according to Kimmel et al. (Kimmel et al., 1995).

\section{Cloning and sequence analysis of EF-2}

A zebrafish cDNA clone RK115A2C01 containing the EF-2 fulllength cDNA was isolated from the zebrafish adult kidney cDNA library (Song et al., unpublished data). The isolated full-length EF-2cDNA was subcloned into $\mathrm{pBK}-\mathrm{CMV}$ vector and the two inserted enzyme sites were EcoRI and Xhol, respectively. Then the full-length EF-2gene was sequenced. Its DNA and amino acid sequences were analyzed on National Center for Biotechnology Information (NCBI) blast server. 
Multiple alignments were performed with clustalw version 1.82 (http:// www.ebi.ac.uk/clustalw/) as well. The graphic presentations were prepared by software GeneDoc.

\section{Whole-mount in situ hybridization}

To study the expression pattern of the EF-2gene during embryogenesis, the zebrafish embryos were processed for whole-mount in situ hybridization after fixation in 4\% PFA for $24 \mathrm{~h}$ at $4^{\circ} \mathrm{C}$. As described previously, the isolated full-length EF-2cDNA sequence was subcloned into $\mathrm{PBK}-\mathrm{CMV}$ vector, which contains $\mathrm{T} 7$ and T3 promoters. Using T3 and T7 RNA polymerases, the sense and antisense RNA probes were synthesized labelled with digoxigenin (Roche) in vitro, respectively. The whole-mount in situhybridization procedure was carried out as described by Westerfield et al. (Westerfield et al., 1995).

\section{Overexpression of EF-2}

Vector pBK-CMV-EF2 was cut by Xho I and used as transcription template. Finally the capped $E F-2 m R N A$ was transcribed in vitrousing T3 RNA polymerase.

Expressing vector pcDNA3.0-EF2 was constructed as following: $E F$ 2 ORF was cut by Xho I and EcoR I from pBK-CMV-EF2 vector, then inserted into pcDNA3.0 expression vector (bought from Invitrogen company) that was also digested by Xho I and EcoR I. Capped EF-2mRNA and various amounts of expression vector pcDNA3.0-EF2 were injected into each of 1-4 cell stage embryos with a fine glass needle connected with an automatic injector (IM-300, Narishige, Japan), respectively. Phenotypes were observed at $48 \mathrm{hpf}$ (hours post-fertilization). We selected three microinjection amounts of expression vector pcDNA3.0-EF2: $1.0 \mathrm{ng} / \mathrm{embryo}, 0.67 \mathrm{ng} / \mathrm{embryo}$ and $0.53 \mathrm{ng} / \mathrm{embryo}$. And the amount of capped EF-2 mRNA microinjection is $4.5 \mathrm{ng} / \mathrm{embryo}$.

\section{Gene knockdown with morpholino antisense oligo}

Both EF-2gene-specific antisense and sense morpholino oligonucleotides were purchased from Gene Tools LLC (Philomath, OR, USA), as following: anti-MO, 5'-CACCATTTTGACAGATGTTCTTGG-3'; senseMO, 5'-CCAAGAACATCTGTCAAAATGGTG-3'.

Morpholino oligonucleotides were injected into each of 2-8-cell-stage embryos with a fine glass needle connected with an automatic injector (IM-300, Narishige, Tokyo, Japan). The amount of microinjection was about 4.5ng/embryo (Nasevicius et al., 2000). Some anti-MO and sense$\mathrm{MO}$ treated embryos at $48 \mathrm{hpf}$ were fixed, dehydrated, embedded in resin and cross-sectioned to $5-10 \mu \mathrm{m}$ thick with a glass knife. Slides were dyed by $1 \%$ toluidine blue.

\section{Acknowledgments}

This work was supported by National Natural Science Foundation of China (30370719) and the National Basic Research Program of China (973) (2004CB518803). We acknowledge the help of Professor A.M. Meng in offering the $A B$ strain zebrafish used as experiment material and are grateful to Mr. Yin Sai for his help with sections.

\section{References}

CHAMBERS, D.M., PETERS, J. AND ABBOTT, C.M. (1998). The lethal mutation of the mouse wasted (wst) is a deletion that abolishes expression of a tissuespecific isoform of translation elongation factor 1alpha, encoded by the EEf1a2 gene. Proc. Natl. Acad. Sci. USA. 95: 4463 - 4468.

DJE, M.K., MAZABRAUD, A., VIEL, A., MAIRE, M., H DENIS, H., CRAWFORD, E. AND BROWN, D.D. (1990). Three genes under different developmental control encode elongation factor 1 -alpha in Xenopus laevis. Nucleic Acids Res. 18 : $3489-3493$.

HOVEMANN, B., RICHTER, S., WALLDORF, U. AND CZIEPLUCH, C. (1988). Two genes encode related cytoplasmic elongation factors 1 alpha ( $E F-1$ alpha) in Drosophila melanogaster with continuous and stage specific expression. Nucleic Acids Res. 16:3175 - 3194.

KIMMEL, C.B., BALLARD, W.W., KIMMEL, S.R., ULLMANN, B. AND SCHILLING, T.F. (1995). Stages of embryonic development of the zebrafish, Dev. Dyn. 203: $253-310$

KNUDSEN, S.M., FRYDENBERG, J., CLARK, B.F. AND LEFFERS, H. (1993). Tissue-dependent variation in the expression of elongation factor-1 alpha isoforms: isolation and characterisation of a cDNA encoding a novel variant of human elongation-factor 1 alpha. Eur. J. Biochem. 215:549 - 554.

KOHNO, K., UCHIDA, T., OHKUBO, H., NAKANISHI, S., NAKANISHI, T., FUKUI, T., OHTSUKA, E., IKEHARA, M. AND OKADA, Y. (1986). Amino acid sequence of mammalian elongation factor 2 deduced from the cDNA sequence: Homology with GTP-binding proteins. Proc. Natl. Acad. Sci. USA. 83:4978 - 4982.

KRIEG, P.A., VARNUM, S.M., WORMINGTON, W.M. AND MELTON, D.A. (1989). The mRNA encoding elongation factor 1-alpha ( $E F-1$ alpha) is a major transcript at the midblastula transition in Xenopus. Dev. Biol. 133:93 -100.

LASKO, P. (2000). The Drosophila melanogastergenome: translation factors and RNA binding proteins. J. Cel/ Biol. 150: 51 - 56.

MALAVE, T.M. and FORNEY, J.D. (2004). Identification of a developmentally regulated translation elongation factor 2 in Tetrahymena thermophila. Gene. 326: 97 - 105

MENDOZA, A., SERRAMIA, M.J., CAPA, L. AND GARCIA-BUSTOS, J.F.(1999). Translation elongation factor 2 is encoded by a single essential gene in Candida albicans. Gene. 229:183 - 191.

NASEVICIUS, A. AND EKKER, S.C. (2000). Effective targeted gene 'knockdown' in zebrafish. Nature Genetics. 26: $216-220$.

RAO, S. AND BODLEY, J.W. (1996). Expression, purification and characterization of the G domain of Saccharomyces cerevisiae elongation factor 2. Protein Exp. Purif. 8:91 - 96.

SONG, H.D., WU, X.Y., SUN,X.J., ZHOU,Y., LIU,T.X., DENG,M., ZHANG,G.W., SHENG,Y., CHEN,Y., RUAN,Z., JIANG,C.L., FAN,H.Y., ZON,L.I., KANKI,J.P., LOOK, A.T. AND CHEN,Z. Gene Expression Profiling in the Zebrafish Kidney Marrow Tissue. Unpublished.

WESTERFIELD, M. (1995). The Zebrafish Book. University of Oregon Press.

Received: August 2005

Reviewed by Referees: October 2005

Modified by Authors and Accepted for Publication: November 2005 